\title{
Older User Errors in Handheld Touchscreen Devices: To What Extent Is Prediction Possible?
}

\author{
Michael Bradley ${ }^{1,2}$, Patrick Langdon ${ }^{1}$, and P. John Clarkson ${ }^{1}$ \\ ${ }^{1}$ Engineering Design Centre, Department of Engineering, University of Cambridge, \\ Trumpington Street, Cambridge. CB2 1PZ United Kingdom \\ ${ }^{2}$ Product Design and Engineering Department, School of Engineering and Information \\ Sciences, Middlesex University, Snakes Lane, London. N14 4YZ United Kingdom \\ \{mab54,pm124,pjc10\} @eng. cam.ac.uk
}

\begin{abstract}
Touchscreen technology has been shown to offer advantages to older and novice users of digital products, through the relative ease of learning the interaction mechanisms and flexibility of the interface to provide explicit and contextual labelling enabling task sequences to be executed. Interaction problems caused by age related changes in sensory perception, cognition and motor skills are able to be predicted using the Inclusive Design Toolkit, however this technique is unable to predict usage problems caused by lack of prior experience of digital interaction patterns. This paper reports on the 'errors' that older users made in a pilot study using a tablet touchscreen device in the course of completing tasks such as turning the device on, setting an alarm and sending an email. An initial classification of the problems encountered by the users is made and the potential for prediction of such errors is discussed.
\end{abstract}

Keywords: Touchscreen, errors, older users, usability, prior experience.

\section{Introduction}

People who do not engage in the digital world, the digitally excluded, are more likely to be older, female and from a lower socio economic and educational background grouping [1]. Older users are likely to exhibit perceptual, sensory and motor skill degradations which will affect their interactions with technology devices, and in particular technology devices which are new to them, and/or exhibit unfamiliar interaction styles [2]. Differing generations also will have experienced differing interface styles during their impressionable younger years, and this can have an impact on interface expectations and expertise [3].

The proportion of the UK population who are unable to achieve certain interactions due to degradation of perceptual, sensory and motor skill performance can currently be estimated using the Inclusive Design Toolkit's exclusion calculator, by comparison of task difficulty to data collected by the ONS database [4]. For example, the exclusion calculator allows for the effects of a small sized font used on a display to be estimated in terms of the proportion of the population who will be unable to use it due to both age and disability related visual impairment. The calculator is able to predict 
the proportion of the UK adult population excluded through the visual, hearing, thinking, dexterity, reach \& stretch and locomotion demands of the product. However, it does not take into account the prior technology experience of the users and their consequent expectations and familiarity with the wide variety of digital interaction patterns [5], nor of their fluidity of intelligence which affects their ability to accommodate to new ways of operating a device [6]. The fragility of learning of newly acquired declarative and procedural knowledge is also not addressed in the calculator. These effects have a very strong impact on the success or otherwise of the interactions that these users have with products with digital interfaces.

\subsection{Background}

Some digitally excluded people complain that technology is not for them, and hence that they don't want to engage with digital technology [1]. It is hypothesised that for people who don't have much digital 'prior experience', this perception is at least partially true: they do not have the pre-requisites to engage with interfaces that are almost always primarily designed for people with a reasonable level of digital technology knowledge. If a legitimate interaction design aim is to make digital technology accessible to people without the digital technology prior experience, it is important to understand their experiences with current technology interfaces, to see where the lack of knowledge and/or training hinders their goal achievement.

In studies with older low technology literate people using digital technology, the usual user performance measures such as time to task completion are not as important as the ability for the user to be able to make error-free progress to their goal achievement [7]. Error making can reinforce the negative feelings of confusion and stress, and frequently put the device into a state from which the user is unable to recover. Slow steady progress without error making is therefore preferable to quick error prone interactions which might be preferable for other user groups who are able to learn and recover from the erroneous steps more swiftly.

\subsection{Errors}

A major stumbling block for unfamiliar users using digital technology interfaces is when 'errors' are made, and the difficulty of recovering from them. These 'errors' are not the same category of error necessarily as those made by a user who is familiar with an interface or a task, who merely makes a slip in execution of a command. These 'errors' are perhaps closer in nature to those made during the process of 'trial and error' providing information to the user about the mental models required to operate the interface correctly. Although for some older people the concept of 'trial and error' as a mechanism for discovering how to use novel device can be at odds with the belief that 'playing with it will break it', and this can significantly impede the learning process for users holding this belief. Additionally, some errors are not discovered by the users, such as if an email is not sent to the correct address, or an appointment not set on the correct day or time in the schedule which can further disrupt the development of accurate mental models. 


\section{Method}

Ten participants were recruited over the age of 55, (range 58 to 78) nine of whom had limited technology prior experience. After the initial explanations of the study purpose, and their rights during the experiment, they were asked to imagine that they had been sent the test device, a Samsung Galaxy Tab (see Fig. 1 for front view of device) as a present through the post, without instructions, nor was there available assistance to guide their interactions. It was however explained that the device was a kind of combination of computer and mobile phone, as well as the fact that it was a touchscreen device. The participants were asked to perform a selection of the following tasks:

- Turn the device on

- Enter a specific passcode to unlock the device

- Send a short email

- Set an alarm for a specific time

- Search the internet for the name of a friend or relative

- Leave the device in a state as if for half an hour

- Leave the device in a state as if going on holiday for two weeks

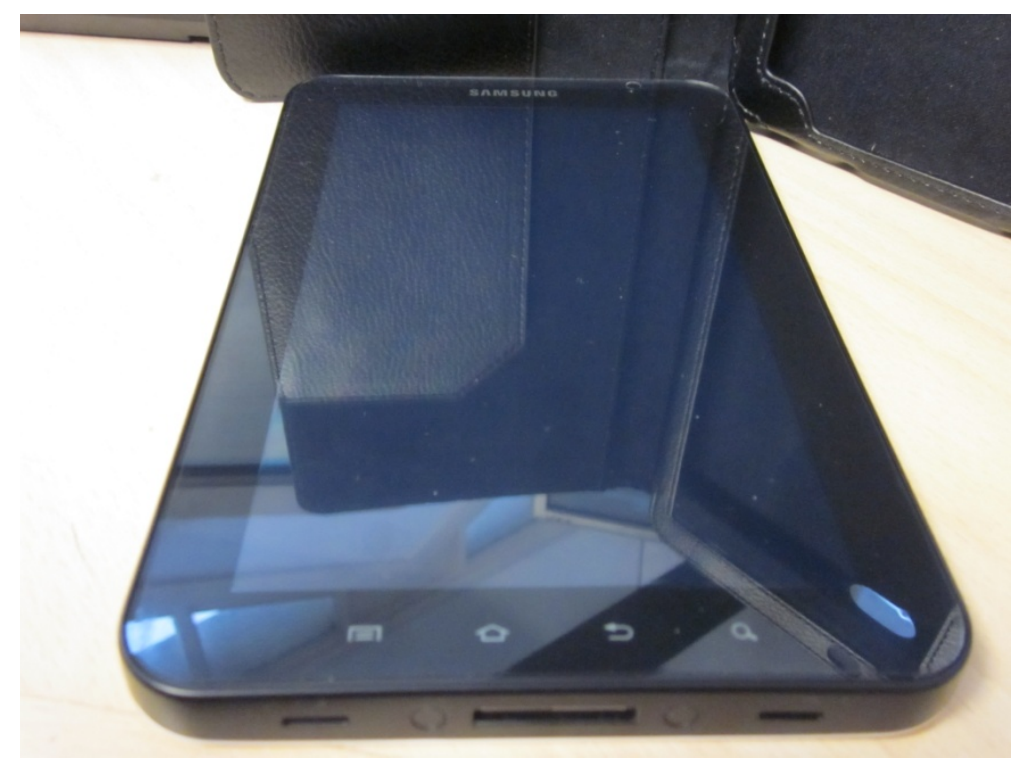

Fig. 1. Samsung Galaxy Tab- Front View Device Off

The participants were asked to explain what they were thinking while they attempted to do the tasks. They were also asked to explain each individual action before they actually performed it, but in practice most of the participants behaved as if they would in a conventional talk aloud protocol. All participants sessions were audio recorded and the experimenter recorded errors made, and five sessions were video recorded for 
more detailed investigation of user actions. The constraints of performing the majority of the sessions in the participants own homes had precluded the use of video for half the participants. Most participants were asked to complete all tasks, however for some less technologically experienced participants the experimenter made the decision that some tasks may tire the participants to the point at which they lose concentration and would not yield useful responses. At the end of their sessions the participants completed technology prior experience questionnaires (modified from Blacker ref) and signed consent forms.

\section{Issues Experienced by Participants}

\subsection{General Issues}

- Not operating the touchscreen reliably - some users inadvertently operated functions they didn't want, and on other occasions wouldn't operate desired functions despite appearing to have performed the correct action (in some cases repeated the correct action a number of times) to do so.

- Some inaccuracy in using the touch keyboard, but more cases of repeated keystrokes on touch keyboard - so two letters appear rather than one.

- Some confusion about how to move the cursor to the desired location - seen in both email and browser searching. A few participants tried to swipe the cursor from its current location to move it to the desired location, which did not appear to work. Others were intent on using the keyboard only to attempt to move it, mainly with the return key, which only succeeded in entering the current data. Some participants needed assistance to get beyond this hurdle.

- Some confusion shown between the concepts of backspace (a left arrow with an $x$ seen on the keyboard) and back (a permanent button below the screen) which were hard for some users to resolve or remember which did what.

\subsection{Issues Relating to Specific Tasks}

Turning device on (see Fig. 2 for image of Power Button):

- Almost all participants struggled for some considerable time to turn the device on, for some or a combination of the following reasons: not being able to see or feel

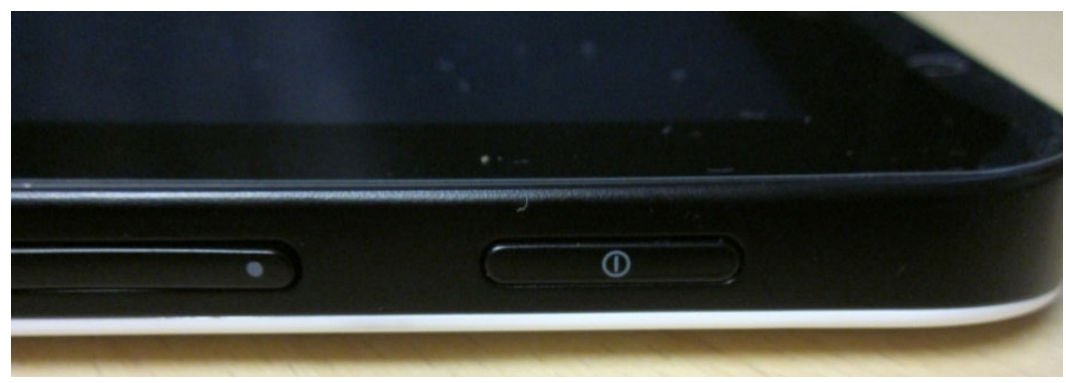

Fig. 2. Samsung Galaxy Tab Side View Showing Power Button Location and Label 
the button at all, not seeing the label on it, or not recognising the on/off button label, if they could see it, in the first instance. Since the button required a long button press to get any response from the device this lack of feedback to a shorter than required press, this contributed to participants eliminating the correct button and focussing their attentions elsewhere. Some even came to the erroneous conclusion that the button required a hard push to work, and in addition one participant said, 'I'm glad I've got fingernails - I wouldn't be able to push this otherwise'.

\section{Entering passcode (See Fig. 3):}

- Most entered number correctly first time, but many struggled to find ' $\mathrm{OK}$ ' button to enter the number. Screen timeouts (in some scenarios only 4 secs before screen timed out and deleted entered numbers) caused much frustration.

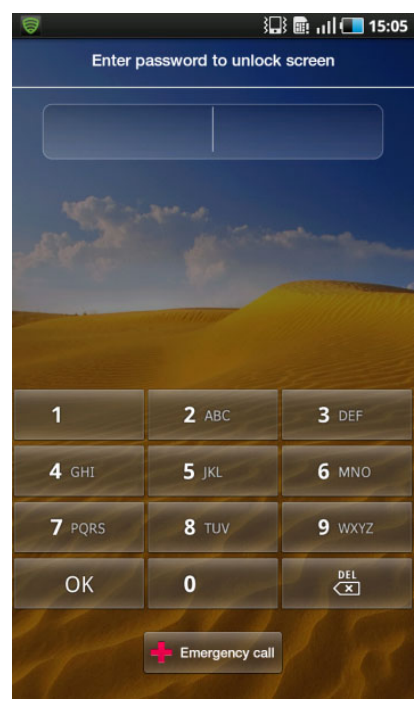

Fig. 3. Passcode Entry Screenshot

\section{Sending email (See Fig. 4):}

- One participant thought that the 'Email' button label was a verb, and therefore that something would need to be created prior to execution of the 'to email' function.

- Not recognising or understanding the function of the 'new compose email' icon/button, or as one user described it as a 'sausage in a grate'.

- No users detected the fleetingly displayed 'Sending...' notification shown at the top of the screen - so none were confident it had been sent.

- One participant correctly entered an email address, and in his attempts to move the cursor to the 'Subject' field by pressing the return key, invoked the device to verify that the email address was of the correct form. The feedback to the user that this had happened, and that the email address was accepted by the system is to put a box around the email address, or if it is a long email address to concatenate and put a box around, such that an email address typed 'longemailaddress@googlemail.com' 
would become 'longemailaddress@go...' in a box displayed on the screen. For one participant this resulted in him re-entering the email address three times to correct the error, before the experimenter intervened.

- Finding an underscore '_, to enter in an email address was found to be impossible for the two participants who tried. The interface requires that either the numerical keyboard is selected using the ' 123 ' key, and then the ' $1 / 3$ ' key (which one participant believed was a thoughtful addition of the one-third key as found on some typewriters) to index the keyboard to the second screen $(2 / 3)$ where the underscore symbol can be found. Alternatively the user presses the '.-;' key for a long button push, and is presented with a submenu of 12 symbols to select, one of which is the underscore.
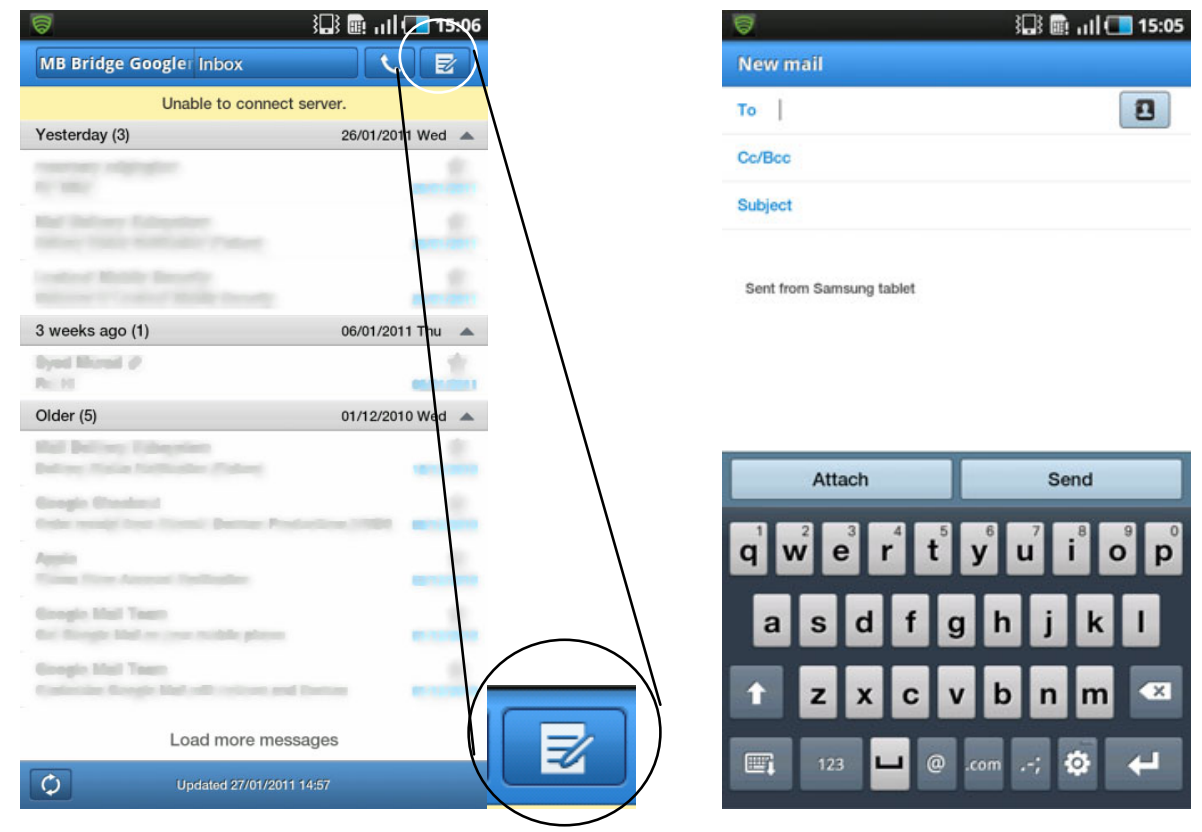

Fig. 4. Email Inbox Screenshot - inset showing Compose New Email Button Detail (Left) and Compose New Email Screenshot (Right)

\section{Search internet:}

- Most users knew Google was an internet search engine so most participants chose one two major pathways - either they selected 'browser' (opening a browser window with Google as home page) or they clicked on the Google search window on the home screen.

- Three participants curiously entered their relative's name (the instruction was to search for a relative or a friend on the internet) without spaces, although Google was in each case able to segregate the names correctly.

- Some participants were sufficiently confused by the appearance of the previous searches using Google in the space beneath the search field that they assumed that they needed to correct it before being able to carry out their desired search. 


\section{Set alarm:}

- A few participants struggled to find alarm clock function (it is located within the 'Applications' menu).

- Many struggled to add an alarm - touching the clock face allows user to alter its appearance but takes away the ability to set the alarm (see Fig. 5). This can be revoked by using the 'back' key or by pressing the 'Set clock style' button, but some participants believed that button would take them to a further menu of options relating to clock styles.

- Once participants had found where to set the alarm time, actually setting the time caused few difficulties (see Fig 5.), but feedback to correct activation of alarm caused many participants confusion due to its ambiguousity.
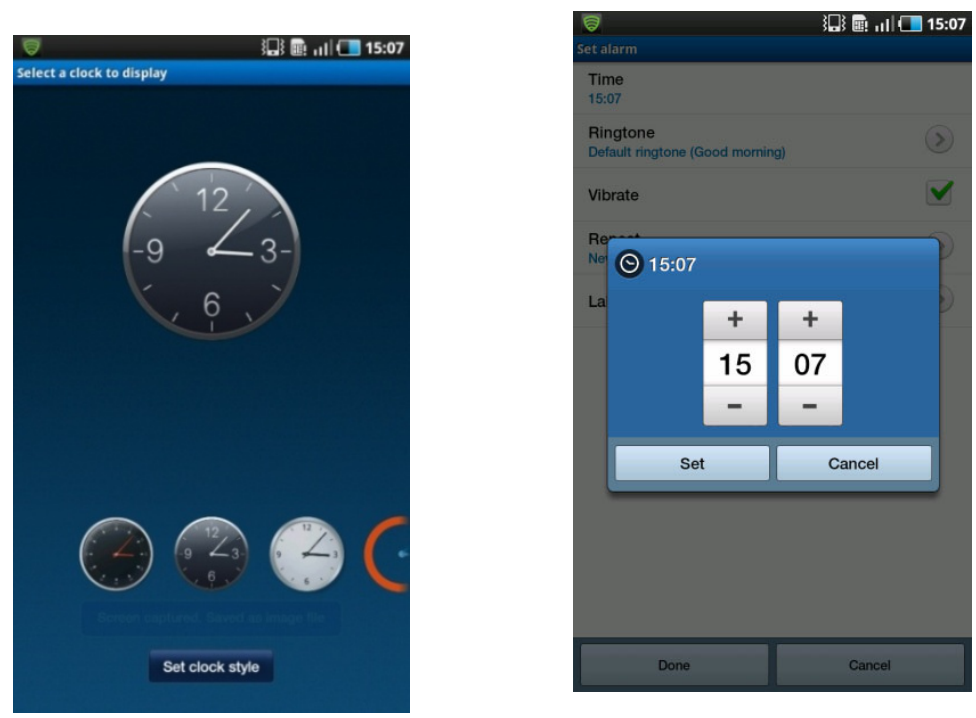

Fig. 5. Clock Face Personalisation (Left) and Alarm Time Setting (Right) Screenshots

\section{Turning off/standby:}

- Many users pressed the power button but not necessarily for the correct length of time (short press invokes standby, long press brings up Phone options menu with 'Power off' option, with then a further confirmation dialogue box). There was some confusion about the difference between off and standby, as well as one participant demonstrating antipathy towards the concept of standby; 'I don't have anything on standby... I can't bear those winking lights... I'd rather have it either off or on'.

\section{Discussion and Conclusion}

An initial classification of the participants' issues put them four categories within two perspectives: User side - those characteristics of the user which contributed to the 
issue experienced, and the Device side - the characteristics of the device which also contributed to the issue (See Table 1). This view permits the comparison and a potentially useful discussion to be had as to how to resolve the issue - whether through redesign of the interface, or perhaps through training to alter the characteristics of the user where appropriate, or indeed, possible.

Table 1. Initial Assessment of Error Causation Classification

\begin{tabular}{|l|l|l|l|l|l|}
\hline $\begin{array}{l}\text { User } \\
\text { characteristics }\end{array}$ & $\begin{array}{l}\text { Lack of } \\
\text { prior } \\
\text { experience }\end{array}$ & Poor vision & $\begin{array}{l}\text { Poor } \\
\text { muscle } \\
\text { control }\end{array}$ & $\begin{array}{l}\text { Searching } \\
\text { strategy } \\
\text { not } \\
\text { optimal }\end{array}$ & $\begin{array}{l}\text { Prior } \\
\text { experience } \\
\text { hinders and } \\
\text { conflicts }\end{array}$ \\
\hline & $\begin{array}{l}\text { Lack of } \\
\text { clarity of } \\
\text { label } \\
\text { meaning } \\
\text { or overly } \\
\text { codified } \\
\text { characteristics }\end{array}$ & $\begin{array}{l}\text { Labelling or } \\
\text { identification } \\
\text { characteristics } \\
\text { too small }\end{array}$ & $\begin{array}{l}\text { Too } \\
\text { sensitive } \\
\text { or not } \\
\text { sensitive } \\
\text { enough } \\
\text { to } \\
\text { respond }\end{array}$ & $\begin{array}{l}\text { Controls } \\
\text { or } \\
\text { displays } \\
\text { not where } \\
\text { expected }\end{array}$ & $\begin{array}{l}\text { Device } \\
\text { behaves } \\
\text { inappropriately } \\
\text { for the user }\end{array}$ \\
\hline
\end{tabular}

All of the issues experienced by the participants fell into at least one of the classifications. For example the adding of the alarm function was difficult for many participants and this was categorised (on the device side) as 'lack of clarity of label meaning or overly codified', 'controls or displays not where expected' and 'device behaves inappropriately for the user'. For this to be useful for the prediction of older user errors in such devices, it is expected that much investigation would be required to populate the understanding of expected locations of controls or displays and appropriate device behaviours for this group. The lack of clarity of label meaning or overly codified category perhaps could be assessed using heuristics from an expert's perspective; for example it is reasonably predictable that the Compose New Email button codification is going to be problematic for some users, due to its somewhat abstract representation of the pen and lines on paper, which itself is an abstraction for the concept of starting a new email.

Further investigations will be carried out to examine the participants' prior experience with more detailed interaction patterns and technology experiences (e.g. use or skill of using a traditional typewriter), to see if a model can be developed which can link this data to user's expectations of interface appearance and behaviours in a predictable way.

\section{References}

[1] Milner, H. (ed.): Does the internet improve lives? UK Online \& Freshminds (2009)

[2] Morris, A., Goodman, J., Brading, H.: Internet use and non-use: views of older users. In: Universal Access in the Information Society, vol. 6(1), pp. 43-57. Springer, Heidelberg (2006)

[3] Docampo Rama, M.: Technology Generations Handling Complex Interfaces, PhD Thesis Eindhoven University of Technology (2001) 
[4] Waller, S., Williams, E., Langdon, P.: Quantifying exclusion for tasks related to product interaction. In: Langdon, P., Clarkson, P.J., Robinson, P. (eds.) Designing Inclusive Interactions, pp. 57-68. Springer, London (2010)

[5] Langdon, P., Hurtienne, J.: Is Prior Experience the same as Intuition in the context of Inclusive Design? In: Langdon, P., Hurtienne, J. (eds.) HCI 2009 Electronic Proceedings: WS4 Prior Experience, British Computer Society, Cambridge (2009)

[6] Czaja, S.J., Lee, C.C.: The impact of aging on access to technology. Universal Access in the Information Society 5(4), 341-349 (2007)

[7] Hawthorn, D.: Designing Effective Interfaces for Older Users, PhD Thesis University of Waikato (2006) 